\title{
Tame Class Field Theory for Singular Varieties over Finite Fields
}

\author{
Thomas Geisser $^{1}$ and Alexander Schmidt ${ }^{2}$
}

March 29, 2022

\section{Introduction}

In the 1980's, Kato and Saito (based on ideas of Bloch) generalized the class field theory for smooth, projective curves over finite fields to smooth, projective varieties of arbitrary dimension [KaSa]: The map from the free abelian group generated by the closed points which sends a generator $x \in X$ to the image of the Frobenius of $k(x)$ under $\pi_{1}^{\mathrm{ab}}(k(x)) \rightarrow \pi_{1}^{\mathrm{ab}}(X)$ factors through the Chow group of zero cycles, and induces an isomorphism

$$
r_{X}: \mathrm{CH}_{0}(X) \stackrel{\sim}{\longrightarrow} \pi_{1}^{\mathrm{ab}}(X)_{\mathrm{W}} .
$$

Here $\pi_{1}^{\mathrm{ab}}(X)_{\mathrm{W}}$ is the subgroup of elements in $\pi_{1}^{\mathrm{ab}}(X)$ whose images in the absolute Galois group of the finite base field are integral Frobenius powers.

If $X$ is not necessarily projective but still smooth, then Schmidt and Spieß SS, Sc] showed that the same result still holds if one replaces the Chow group by Suslin homology and the fundamental group by its tame version: The reciprocity map induces an isomorphism of finitely generated abelian groups

$$
r_{X}: H_{0}^{\mathrm{S}}(X, \mathbb{Z}) \stackrel{\sim}{\longrightarrow} \pi_{1}^{t, \mathrm{ab}}(X)_{\mathrm{W}} .
$$

This result does not extend to non-smooth schemes: the example of the node shows that $r_{X}$ is neither injective nor surjective in general. If $X$ is normal, then $r_{X}$ is surjective but an example of Matsumi-Sato-Asakura [MAS] shows that $r_{X}$ may have a nontrivial kernel.

In this paper, we show that the result of Schmidt and Spieß can be generalized to singular varieties if one uses a refined version of Suslin homology on the one hand, and replaces the fundamental group by the enlarged fundamental group of SGA3. $\mathrm{X}, \S 6]$ on the other hand. We denote the abelian enlarged tame fundamental group by $\Pi_{1}^{t, a b}$ in order to distinguish it from the usual abelian tame fundamental group $\pi_{1}^{t, \mathrm{ab}}$, which is its profinite completion. The groups coincide if $X$ geometrically unibranch (e.g., normal). Our first result is:

Theorem 1.1. For any connected scheme $X$, separated and of finite type over a finite field, the pro-group $\Pi_{1}^{t, \mathrm{ab}}(X)_{\mathrm{W}}$ is isomorphic to a (constant) finitely generated abelian group.

On the other hand, recall from Ge3 the definition of Weil-Suslin homology: Let $\mathbb{F}$ be the finite base field, $F \in \mathrm{Gal}_{\mathbb{F}}$ be the Frobenius automorphism and $G \cong \mathbb{Z}$ the

\footnotetext{
${ }^{1}$ Supported by JSPS Grant-in-Aid (B) 23340004

${ }^{2}$ Supported by DFG-Forschergruppe FOR 1920
} 
subgroup of $\mathrm{Gal}_{\mathbb{F}}$ generated by $F$. For an abelian group $A$, the groups $H_{i}^{\mathrm{WS}}(X, A)$ are defined as the homology of the cone of $1-F^{*}$ on the Suslin complex tensored by $A$ of the base change $\bar{X}$ of $X$ to the algebraic closure $\overline{\mathbb{F}}$ of $\mathbb{F}$. By definition, there are short exact sequences

$$
0 \longrightarrow H_{i}^{\mathrm{S}}(\bar{X}, A)_{G} \longrightarrow H_{i}^{\mathrm{WS}}(X, A) \longrightarrow H_{i-1}^{\mathrm{S}}(\bar{X}, A)^{G} \longrightarrow 0 .
$$

Furthermore, there are natural maps for all $i$

$$
H_{i}^{\mathrm{S}}(X, A) \longrightarrow H_{i+1}^{\mathrm{WS}}(X, A) .
$$

If $X$ is smooth and $A$ is finite, then it follows from the proof of Kato's conjecture by Jannsen, Kerz and Saito KeSa (and under resolution of singularities) that these maps are isomorphisms (for $i=0$ this follows from the theorem of Schmidt-Spieß).

We define a refined reciprocity homomorphism

$$
\operatorname{rec}_{X}: H_{1}^{\mathrm{WS}}(X, \mathbb{Z}) \longrightarrow \Pi_{1}^{t, \mathrm{ab}}(X)_{\mathrm{W}}
$$

such that the composite with the natural map $H_{0}^{\mathrm{S}}(X, \mathbb{Z}) \rightarrow H_{1}^{\mathrm{WS}}(X, \mathbb{Z})$ is the reciprocity map $r_{X}$ described above. Our main result, conjectured in Ge3], is the following

Theorem 1.2. For any connected scheme $X$, separated and of finite type over a finite field $\mathbb{F}$, the homomorphism

$$
\operatorname{rec}_{X}: H_{1}^{\mathrm{WS}}(X, \mathbb{Z}) \longrightarrow \Pi_{1}^{t, \mathrm{ab}}(X)_{\mathrm{W}}
$$

is surjective. The kernel of $\operatorname{rec}_{X}$ contains, and if resolution of singularities for schemes of dimension $\leq \operatorname{dim} X+1$ holds over $\mathbb{F}$ is equal to, the maximal divisible subgroup of $H_{1}^{\mathrm{WS}}(X, \mathbb{Z})$.

As a corollary, we obtain (under resolution of singularities) an isomorphism of profinite completions

$$
\operatorname{rec}_{X}^{\wedge}: H_{1}^{\mathrm{WS}}(X, \mathbb{Z})^{\wedge} \stackrel{\sim}{\longrightarrow} \pi_{1}^{t, \mathrm{ab}}(X) .
$$

Under Parshin's conjecture (cf. Ge3] $), H_{1}^{\mathrm{WS}}(X, \mathbb{Z})$ is finitely generated, hence rec $_{X}$ would be an isomorphism.

\section{The fundamental group and tame coverings}

\subsection{Etale and Weil-etale cohomology}

Let $X$ be a scheme, separated and of finite type over a finite field $\mathbb{F}$. The absolute Galois group $\mathrm{Gal}_{\mathbb{F}}$ acts on $\bar{X}=X \times_{\mathbb{F}} \overline{\mathbb{F}}$ via its action on $\overline{\mathbb{F}}$. Pulling an etale sheaf $\mathcal{F}$ on $X$ back to $\overline{\mathcal{F}}$ on $\bar{X}$, we obtain a sheaf with a Gal ${ }_{\mathbb{F}}$-action, cf. SGA7, XIII $\S 1.1]$. Using this action, the etale cohomology $H_{\mathrm{et}}^{*}(X, \mathcal{F})$ can be calculated as the cohomology of $R \Gamma_{\mathrm{Gal}_{\mathbb{F}}} R \Gamma_{\text {et }}(\bar{X}, \overline{\mathcal{F}})$. The Weil-etale cohomology of $\mathcal{F}$ is by definition the cohomology of $R \Gamma_{G} R \Gamma_{\text {et }}(\bar{X}, \overline{\mathcal{F}})$, where $G \cong \mathbb{Z}$ is the subgroup of Gal $\mathbb{F}_{\mathbb{F}}$ generated by the Frobenius. Since $R \Gamma_{\mathrm{Gal}_{\mathbb{F}}}$ and $R \Gamma_{G}$ coincide on discrete torsion modules, etale and Weil-etale cohomology coincide on torsion sheaves (cf. [Ge1, §2] for a more detailed account).

We can calculate the Weil-etale cohomology of a sheaf $\mathcal{F}$ as follows: Choose an injective resolution $\mathcal{F} \rightarrow \mathrm{I}^{\bullet}$. Then

$$
H_{\mathrm{et}}^{i}(X, \mathcal{F})=H^{i}\left(\mathrm{I}^{\bullet}(\bar{X})^{G}\right),
$$


and

$$
H_{\mathrm{W}}^{i}(X, A)=H^{i}\left(\mathrm{I}^{\bullet}(\bar{X}) \stackrel{1-F^{*}}{\longrightarrow} \mathrm{I}^{\bullet}(\bar{X})\right),
$$

where $F^{*}$ is the pull-back along the Frobenius, and the left complex is considered to be in homological degree 0 . We form the double complex by using the negative of the differential in the second complex, i.e., the differential of the total complex has the form

$$
\mathrm{I}^{i}(\bar{X}) \oplus \mathrm{I}^{i-1}(\bar{X}) \rightarrow \mathrm{I}^{i+1}(\bar{X}) \oplus \mathrm{I}^{i}(\bar{X}),(\alpha, \beta) \mapsto\left(d \alpha, \alpha-F^{*} \alpha-d \beta\right) .
$$

From the definition, we obtain short exact sequences

$$
0 \longrightarrow H_{\mathrm{et}}^{i-1}(\bar{X}, \mathcal{F})_{G} \longrightarrow H_{\mathrm{W}}^{i}(X, \mathcal{F}) \longrightarrow H_{\mathrm{et}}^{i}(\bar{X}, \mathcal{F})^{G} \longrightarrow 0,
$$

as well as a homomorphism

$$
H_{\mathrm{et}}^{i}(X, \mathcal{F}) \longrightarrow H_{\mathrm{W}}^{i}(X, \mathcal{F})
$$

for each $i \geq 0$, which is an isomorphism for $i=0$ and injective for $i=1$.

By [SV1, Thm. 10.2], etale and qfh-cohomology of a constant sheaf $A$ coincide. Hence, in order to calculate Weil-etale cohomology of $A$, we can also work with an injective resolution $A \rightarrow \mathrm{I}^{\bullet}$ in the big qfh-site over $\mathbb{F}$. If moreover $A$ is a $\mathbb{Z} / m$ module for some $m \geq 1$, then we can also work with a resolution of $A$ by injective $h$-sheaves of $\mathbb{Z} / m$-modules, see [SV1, Thm. 10.7].

For a regular connected curve $C$ over a field $k$ we consider the subgroup $H_{t}^{1}(C, A)$ $\subseteq H_{\mathrm{et}}^{1}(C, A)$ of tame cohomology classes (corresponding to those continuous homomorphisms $\pi_{1}^{\text {et }}(C) \rightarrow A$ which factor through the tame fundamental group $\pi_{1}^{t}\left(C^{\prime}, C^{\prime}-C\right)$, where $C^{\prime}$ is the regular compactification of $\left.C\right)$.

For a general $k$-scheme $X$, we call a cohomology class in $a \in H_{\mathrm{et}}^{1}(X, A)$ tame if for any morphism $f: C \rightarrow X$ with $C$ a regular curve, we have $f^{*}(a) \in H_{t}^{1}(C, A)$. The tame cohomology classes form a subgroup

$$
H_{t}^{1}(X, A) \subseteq H_{\mathrm{et}}^{1}(X, A) .
$$

The groups coincide if $X$ is proper, if $p=0$, or if $p>0$ and $A$ is $p$-torsion free, where $p$ is the characteristic of the base field $k$. If $X$ is smooth with smooth compactification $X^{\prime}$, then $H_{t}^{1}\left(X, \mathbb{Z} / p^{r}\right) \cong H_{\text {et }}^{1}\left(X^{\prime}, \mathbb{Z} / p^{r}\right)$ for any $r \geq 1$ by GS, Prop. 2.10].

For $X$ separated and of finite type over the finite field $\mathbb{F}$, we define the tame Weil-etale cohomology to be the subgroup

$$
H_{\mathrm{W}, t}^{1}(X, A) \subseteq H_{\mathrm{W}}^{1}(X, A)
$$

of those elements, whose image in $H_{\mathrm{et}}^{1}(\bar{X}, A)$ in (2) is tame.

Recall that an abstract blow-up square is a cartesian diagram

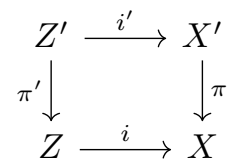

such that $i$ is a closed embedding, $\pi$ is proper, and $\pi$ induces an isomorphism $\left(X^{\prime}-Z^{\prime}\right)_{\text {red }} \stackrel{\sim}{\rightarrow}(X-Z)_{\text {red }}$. 
Proposition 2.1. If in the abstract blow-up square (4) $\pi$ is finite, or if the abelian group $A$ is torsion, then there is an exact sequence

$$
\begin{aligned}
0 \longrightarrow H_{\mathrm{W}}^{0}(X, A) & \longrightarrow H_{\mathrm{W}}^{0}\left(X^{\prime}, A\right) \oplus H_{\mathrm{W}}^{0}(Z, A) \longrightarrow H_{\mathrm{W}}^{0}\left(Z^{\prime}, A\right) \\
& \longrightarrow H_{\mathrm{W}, t}^{1}(X, A) \longrightarrow H_{\mathrm{W}, t}^{1}\left(X^{\prime}, A\right) \oplus H_{\mathrm{W}, t}^{1}(Z, A) \longrightarrow H_{\mathrm{W}, t}^{1}\left(Z^{\prime}, A\right) .
\end{aligned}
$$

Proof. Let Sch $/ \mathbb{F}$ be the category of separated schemes of finite type over $\mathbb{F}$. For $S \in \mathrm{Sch} / \mathbb{F}$ we denote by $\mathbb{Z}_{h}(S)$ the $h$-sheaf of abelian groups associated with the presheaf defined by $U \mapsto \mathbb{Z}\left[\operatorname{Mor}_{\mathbb{F}}(U, S)\right]$.

For any finite field extension $\mathbb{F}^{\prime} / \mathbb{F}$, the base changes of $X, Z, Z^{\prime}, X^{\prime}$ to $\mathbb{F}^{\prime}$ form an abstract blow-up square in a natural way. By the same argument as in the proof of [Ge2, Prop. 3.2] (for the eh-topology) or [SV2, Lem. 12.1] (for the cdh-topology), we have an exact sequence of $h$-sheaves on $\mathrm{Sch} / \mathbb{F}$

$$
0 \rightarrow \mathbb{Z}_{h}\left(Z_{\mathbb{F}^{\prime}}^{\prime}\right) \rightarrow \mathbb{Z}_{h}\left(Z_{\mathbb{F}^{\prime}}\right) \oplus \mathbb{Z}_{h}\left(X_{\mathbb{F}^{\prime}}^{\prime}\right) \rightarrow \mathbb{Z}_{h}\left(X_{\mathbb{F}^{\prime}}\right) \rightarrow 0
$$

If $A$ is torsion, étale and $h$-cohomology with values in $A$ agree by [SV1, Thm.10.2]. Applying the functor $R \operatorname{Hom}_{h}(-, A)$ and passing to the limit over all $\mathbb{F}^{\prime} / \mathbb{F}$, we obtain the exact triangle

$$
R \Gamma\left(\bar{X}_{\mathrm{et}}, A\right) \rightarrow R \Gamma\left(\bar{X}_{\mathrm{et}}^{\prime}, A\right) \oplus R \Gamma\left(\bar{Z}_{\mathrm{et}}, A\right) \rightarrow R \Gamma\left(\bar{Z}_{\mathrm{et}}^{\prime}, A\right) \rightarrow R \Gamma\left(\bar{X}_{\mathrm{et}}, A\right)[1]
$$

and the long exact sequence

$$
\rightarrow H_{\mathrm{et}}^{i}(\bar{X}, A) \rightarrow H_{\mathrm{et}}^{i}\left(\bar{X}^{\prime}, A\right) \oplus H_{\mathrm{et}}^{i}(\bar{Z}, A) \rightarrow H_{\mathrm{et}}^{i}\left(\bar{Z}^{\prime}, A\right) \rightarrow H_{\mathrm{et}}^{i+1}(\bar{X}, A) \rightarrow .
$$

If $\pi$ is finite, we have the qfh-version of the exact sequence (5) and obtain (6) and (7) for arbitrary $A$, since etale and qfh-cohomology with values in any abelian group agree by [SV1, Thm. 10.7]. Applying $R \Gamma_{G}$ to (6) and taking cohomology, we obtain the long exact sequence

$$
H_{\mathrm{W}}^{i}(X, A) \rightarrow H_{\mathrm{W}}^{i}\left(X^{\prime}, A\right) \oplus H_{\mathrm{W}}^{i}(Z, A) \rightarrow H_{\mathrm{W}}^{i}\left(Z^{\prime}, A\right) \rightarrow H_{\mathrm{W}}^{i+1}(X, A) \rightarrow \cdots .
$$

By [GS, Prop. 5.1], (7) induces an exact sequence

$$
\begin{aligned}
0 \longrightarrow H_{\mathrm{et}}^{0}(\bar{X}, A) & \longrightarrow H_{\mathrm{et}}^{0}\left(\bar{X}^{\prime}, A\right) \oplus H_{\mathrm{et}}^{0}(\bar{Z}, A) \longrightarrow H_{\mathrm{et}}^{0}\left(\bar{Z}^{\prime}, A\right) \\
& \stackrel{\delta}{\longrightarrow} H_{t}^{1}(\bar{X}, A) \longrightarrow H_{t}^{1}\left(\bar{X}^{\prime}, A\right) \oplus H_{t}^{1}(\bar{Z}, A) \longrightarrow H_{t}^{1}\left(\bar{Z}^{\prime}, A\right) .
\end{aligned}
$$

Comparing (9) with the sequences (7) and (8), we obtain the statement of the proposition by a diagram chase.

\subsection{The enlarged fundamental group}

We recall the definition of the enlarged fundamental group of [SGA3, X §6]: Let $X$ be a connected, locally noetherian scheme. For a group $G$ (considered as a constant group scheme over $X$ ), a $G$-torsor $P$ over $X$ is a non-empty etale $X$-scheme $P$ (i.e., $\pi: P \rightarrow X$ is unramified, flat and locally of finite type) with a $G$-action $P \times_{X} G \rightarrow P$ such that $P \times_{X} G \rightarrow P \times_{X} P,(x, g) \mapsto(x, x g)$, is an isomorphism. By [Mi, Prop. 2.7] (see also Ex. 2.6, loc. cit.), for any etale sheaf $F$ on $X$ we have a Hochschild-Serre spectral sequence

$$
E_{2}^{r s}=H^{r}\left(G, H_{\mathrm{et}}^{s}\left(P, \pi^{*} F\right)\right) \Longrightarrow H_{\mathrm{et}}^{r+s}(X, F) .
$$

For a geometric point $\xi$ of $X$, one defines $\Pi^{1}(X, \xi, G)$ to be the set of isomorphism classes of $G$-torsors over $X$ pointed over $\xi$. The trivial $G$-torsor gives a distinguished 
element in $\Pi^{1}(X, \xi, G)$. For a $G$-torsor $P$ on $X$ and a group homomorphism $f$ : $G \rightarrow H$, consider

$$
f_{*}(P):=(P \times H) / G
$$

where $G$ acts by $(t, h) \cdot g=\left(t g, f\left(g^{-1}\right) h\right)$. Then $f_{*}(P)$ is an $H$-torsor over $X$ and we obtain a functor

$$
G \longmapsto \Pi^{1}(X, \xi, G)
$$

from groups to pointed sets. By [SGA3, X $\S 6]$, this functor is pro-represented by the enlarged fundamental pro-group $\Pi_{1}(X, \xi)$, i.e.,

$$
\Pi^{1}(X, \xi, G) \cong \operatorname{Hom}_{\text {pro-grps }}\left(\Pi_{1}(X, \xi), G\right) .
$$

Explicitly, there is a pro-system of groups $\Pi_{1}(X, \xi)=\left(G_{i}\right)_{i \in I}$ with $I$ filtering, and a $G_{i}$-torsor $P_{i}$ corresponding to the projection map $\Pi_{1}(X, \xi) \rightarrow G_{i}$ for all $i$ such that for any transition map $\alpha_{i j}: G_{i} \rightarrow G_{j}$ in the system we have $P_{j}=\left(\alpha_{i j}\right)_{*}\left(P_{i}\right)$, and such that for any morphism $\Pi_{1}(X, \xi) \rightarrow H$ represented by $f: G_{j} \rightarrow H$ in the filtered colimit, the corresponding $H$-torsor is $f_{*}\left(P_{j}\right)$.

Next we define the enlarged tame fundamental group by extending the notion of curve-tameness from $\mathrm{KeSc}$ to the enlarged context. A $G$-torsor $P$ over a regular connected curve $C$ over a field $k$ is called tame, if the projection $P \rightarrow C$ extends to an at most tamely ramified covering of the regular compactification $C^{\prime}$ of $C$. If $X$ is any scheme, separated and of finite type over $k$, and $P$ is a $G$-torsor on $X$, then we call $P$ tame if its pull back to the normalization of any curve on $X$ is a tame torsor. If $G=A$ is abelian, then an $A$-torsor is tame if and only if its associated class in $H_{\mathrm{et}}^{1}(X, A)$ lies in $H_{t}^{1}(X, A)$.

The functor

$$
G \longmapsto \Pi^{1, t}(X, \xi, G)
$$

sending $G$ to the set of isomorphism classes of pointed tame $G$-torsors on $X$ is pro-represented by the enlarged tame fundamental group $\Pi_{1}^{t}(X, \xi)$, a quotient of $\Pi_{1}(X, \xi)$ in the category of pro-groups.

The abelianizations $\Pi_{1}^{\mathrm{ab}}(X)$ and $\Pi_{1}^{t, \mathrm{ab}}(X)$ of $\Pi_{1}(X, \xi)$ and $\Pi_{1}^{t}(X, \xi)$ represent the restrictions of the respective functors to the category of abelian groups and are independent of the chosen base point $\xi$.

Lemma 2.2. For any abelian group $A$ we have

$$
\operatorname{Hom}_{\text {pro-grps }}\left(\Pi_{1}^{\mathrm{ab}}(X), A\right) \cong H_{\mathrm{et}}^{1}(X, A)
$$

and similarly for the tame version.

Proof. Write $\Pi_{1}^{\mathrm{ab}}(X)=\left(A_{i}\right)$ and $P_{i}$ for the $A_{i}$-torsor corresponding to the projection $\Pi_{1}^{\mathrm{ab}}(X) \rightarrow A_{i}$. We obtain a filtered direct system of Hochschild-Serre spectral sequences

$$
H^{r}\left(A_{i}, H_{\mathrm{et}}^{s}\left(P_{i}, A\right)\right) \Rightarrow H_{\mathrm{et}}^{r+s}(X, A)
$$

inducing a system of short exact sequences

$$
0 \longrightarrow H^{1}\left(A_{i}, A\right) \longrightarrow H_{\mathrm{et}}^{1}(X, A) \longrightarrow H^{0}\left(A_{i}, H_{\mathrm{et}}^{1}\left(P_{i}, A\right)\right) .
$$

The right map is the zero map in the colimit over all $i$, because if the $A$-torsor $P$ arises from a map $f: A_{i} \rightarrow A$, i.e., $P=f_{*}\left(P_{i}\right)$, then $P$ trivializes over $P_{i}$. Finally,

$$
\operatorname{colim} H^{1}\left(A_{i}, A\right) \cong \operatorname{colim} \operatorname{Hom}\left(A_{i}, A\right)=\operatorname{Hom}_{\text {pro-grps }}\left(\Pi_{1}^{\mathrm{ab}}(X), A\right) \text {. }
$$

From now on let $X$ be a connected scheme, separated and of finite type over the finite field $\mathbb{F}$. As above, we denote the subgroup of $\mathrm{Gal}_{\mathbb{F}}$ consisting of integral powers of the Frobenius automorphism by $G$. 
Definition 2.3. The enlarged Weil-tame fundamental group $\Pi_{1}^{t}(X, \xi)_{\mathrm{W}}$ is defined by the cartesian diagram of pro-groups

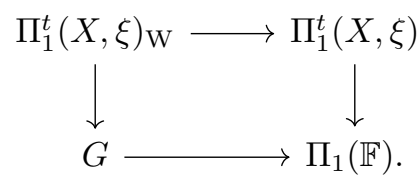

The abelianization $\Pi_{1}^{t, \mathrm{ab}}(X)_{\mathrm{W}}$ of $\Pi_{1}^{t}(X, \xi)_{\mathrm{W}}$ fits into the analogous cartesian diagram

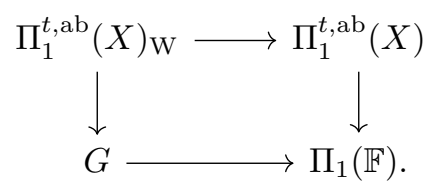

The profinite completion of $\Pi_{1}^{t, \mathrm{ab}}(X)_{\mathrm{W}}$ is the usual abelian (curve-)tame fundamental group $\pi_{1}^{t, a b}(X)$ of $[\mathrm{KeSc}]$.

Proposition 2.4. For any abelian group $A$, there is a functorial isomorphism

$$
\operatorname{Hom}_{\text {pro-grps }}\left(\Pi_{1}^{\mathrm{ab}}(X)_{\mathrm{W}}, A\right) \cong H_{\mathrm{W}}^{1}(X, A)
$$

compatible with the isomorphism of Lemma 2.2. and there is a similar isomorphism for the tame version.

Proof. Replacing $\mathbb{F}$ by its maximal algebraic extension in $\Gamma\left(X, \mathcal{O}_{X}\right)$ changes $G$ to a subgroup of finite index, but does not change the groups on both sides of the statement. Hence we may assume that $X$ is geometrically connected DG, I, $\S 4$, 6.7]. Setting $\bar{X}=X \times_{\mathbb{F}} \overline{\mathbb{F}}$, we have the exact sequence of pro-groups

$$
1 \longrightarrow \Pi_{1}(\bar{X}, \xi) \longrightarrow \Pi_{1}(X, \xi)_{\mathrm{W}} \longrightarrow G \longrightarrow 1 .
$$

If we write $\Pi_{1}(X, \xi)_{\mathrm{W}}=\left(G_{i}\right)$, then $\Pi_{1}(\bar{X}, \xi)=\left(\bar{G}_{i}\right)$ with $\bar{G}_{i}=\operatorname{ker}\left(G_{i} \rightarrow G\right)$. We denote the $\bar{G}_{i}$-torsor over $\bar{X}$ associated with the projection map $\Pi_{1}(\bar{X}, \xi) \rightarrow \bar{G}_{i}$ by $\bar{P}_{i}$.

Consider the functor $\mathcal{F} \mapsto \Gamma\left(\bar{P}_{i}, \mathcal{F}\right)$ from the category of etale sheaves on $X$ to $G_{i}$-modules. The inclusion $A \rightarrow R \Gamma\left(\bar{P}_{i}, A\right)$ induces a map $R \Gamma_{G_{i}}(A) \rightarrow$ $R \Gamma_{G_{i}} R \Gamma\left(\bar{P}_{i}, A\right)$ in the derived category of abelian groups. Since $R \Gamma_{G_{i}}=R \Gamma_{G} R \Gamma_{\bar{G}_{i}}$, we can write this map in the form

$$
R \Gamma_{G_{i}}(A) \longrightarrow R \Gamma_{G} R \Gamma_{\bar{G}_{i}} R \Gamma\left(\bar{P}_{i}, A\right) .
$$

Since taking global sections over $\bar{P}_{i}$ has an exact left adjoint, it sends injectives sheaves on $X$ to injective $\mathbb{Z} \bar{G}_{i}$-modules, and we obtain $R \Gamma_{\bar{G}_{i}} R \Gamma\left(\bar{P}_{i}, A\right)=R \Gamma(\bar{X}, A)$. We thus can write (10) in the form

$$
R \Gamma_{G_{i}}(A) \longrightarrow R \Gamma_{G} R \Gamma(\bar{X}, A) .
$$

Taking cohomology, and passing to the colimit over $i$, we obtain maps

$$
H^{n}\left(\Pi_{1}^{\mathrm{ab}}(X)_{\mathrm{W}}, A\right) \longrightarrow H_{\mathrm{W}}^{n}(X, A), \quad n \geq 0 .
$$

For $n=1$ this is the map of the proposition and we have to show that it is an isomorphism. For this we rewrite (11) in the form

$$
R \Gamma_{G} R \Gamma_{\bar{G}_{i}}(A) \longrightarrow R \Gamma_{G} R \Gamma(\bar{X}, A)
$$


and consider the map of associated spectral sequences, which degenerate to short exact sequences. In degree 1 , we obtain the commutative diagram with exact lines

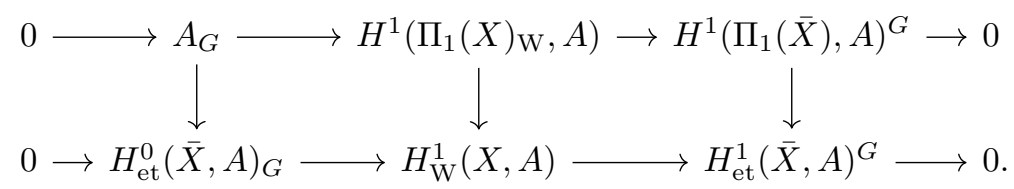

Since $X$ is geometrically connected, the left hand vertical map is an isomorphism. The right hand vertical map is an isomorphism by Lemma 2.2. Hence the middle map is an isomorphism.

To show the statement for the tame variant, we note that there is a similar diagram as above for the tame groups. Indeed, a torsor on $X$ is tame if and only if its base change to $\bar{X}$ is tame, so we obtain the exact sequence

$$
1 \longrightarrow \Pi_{1}^{t}(\bar{X}, \xi) \longrightarrow \Pi_{1}^{t}(X, \xi)_{\mathrm{W}} \longrightarrow G \longrightarrow 1 \text {. }
$$

On the other hand, the lower sequence of the above diagram induces a similar sequence for the tame Weil-etale cohomology by definition. This time, the right hand vertical map is an isomorphism by the tame version of Lemma 2.2 .

For a finite disjoint union of connected schemes $X=\amalg X_{i}$ we write by abuse of notation

$$
\Pi_{1}^{t, \mathrm{ab}}(X)_{\mathrm{W}}=\prod_{i} \Pi_{1}^{t, \mathrm{ab}}\left(X_{i}\right)_{\mathrm{W}} .
$$

Theorem 2.5. For any $X$, separated and of finite type over a finite field $\mathbb{F}$, $\Pi_{1}^{t, \mathrm{ab}}(X)_{\mathrm{W}}$ is isomorphic to a finitely generated abelian group.

Proof. Let us first assume that $X$ is normal and connected. We claim that the kernel

$$
\Pi_{1}^{t, \mathrm{ab}}(X)^{\mathrm{geo}}:=\operatorname{ker}\left(\Pi_{1}^{t, \mathrm{ab}}(X)_{\mathrm{W}} \longrightarrow \Pi_{1}(\mathbb{F})_{\mathrm{W}} \cong \mathbb{Z}\right)
$$

is a finite abelian group. If $X$ is smooth, this follows from the main theorem of Schmidt-Spieß [SS, Sc]. For a general normal $X$, choose a dense open smooth subscheme $U \subset X$. Then, by [SGA1, V, Prop. 8], $\Pi_{1}^{t, \text { ab }}(U)^{\text {geo }}$ surjects onto $\Pi_{1}^{t, \text { ab }}(X)^{\text {geo }}$, hence the latter group is finite.

Now let $X$ be arbitrary. We can assume that $X$ is connected and reduced. Let $X^{\prime} \rightarrow X$ be the normalization. The cokernel $\Pi_{1}^{\mathrm{ab}}\left(X^{\prime} / X\right)$ of $\Pi_{1}^{\mathrm{ab}}\left(X^{\prime}\right) \rightarrow \Pi_{1}^{\mathrm{ab}}(X)$ represents the functor $\Pi_{\mathrm{ab}}^{1}\left(X^{\prime} / X\right)$ which sends an abelian group $A$ to the set of isomorphism classes of $A$-torsors on $X$ which trivialize over $X^{\prime}$. We denote the tame version of this group by $\Pi_{1}^{t, \mathrm{ab}}\left(X^{\prime} / X\right)$ and the cokernel of $\Pi_{1}^{t, \mathrm{ab}}\left(X^{\prime}\right)_{\mathrm{W}} \rightarrow \Pi_{1}^{t, \mathrm{ab}}(X)_{\mathrm{W}}$ by $C$. Consider the diagram

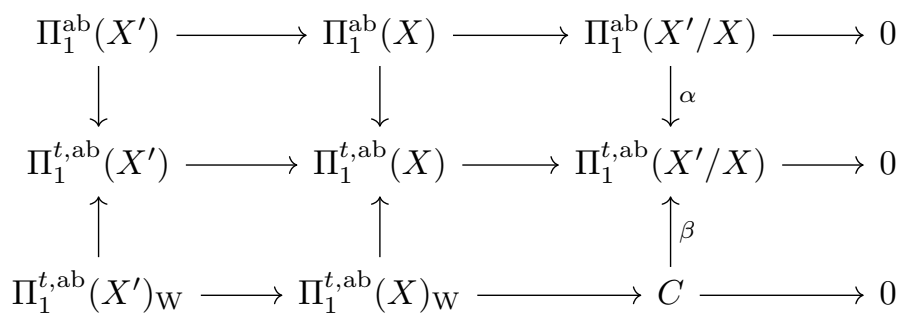

Since $X^{\prime} \rightarrow X$ is proper, a torsor on $X$ which trivializes over $X^{\prime}$ is tame. Hence $\alpha$ is an isomorphism, and so is $\beta$. By [SGA3, $\mathrm{X} \S 6, \mathrm{p} .109], \Pi_{1}^{\mathrm{ab}}\left(X^{\prime} / X\right)$ is a finitely generated abelian group, hence so is $C$. We proved above that the geometric part of $\Pi_{1}^{t, \mathrm{ab}}\left(X^{\prime}\right)_{\mathrm{W}}$ (defined componentwise if $X^{\prime}$ is not connected) is finite. This implies that $\Pi_{1}^{t, a b}(X)_{\mathrm{W}}$ is constant and finitely generated. 


\section{Weil-Suslin homology}

Let $k$ be a perfect field and $X$ a scheme, separated and of finite type over $k$. We recall that, for a smooth $k$-scheme $T$, the group of finite correspondences $\operatorname{Cor}(T, X)$ is the free abelian group generated by closed integral $Z \subseteq T \times X$ which are finite and surjective over a component of $T$. The Suslin complex of $X$ is the complex $C_{\bullet}(X)=\operatorname{Cor}\left(\Delta^{\bullet}, X\right)$, where $\Delta^{i}=\operatorname{Spec} k\left[T_{0}, \ldots, T_{i}\right] /\left(\Sigma T_{i}-1\right)$. Putting $\partial:=\sum_{j=0}^{i}(-1)^{j} \delta_{i}^{j} \in \operatorname{Cor}\left(\Delta^{i-1}, \Delta^{i}\right)$, where $\delta_{i}^{j}: \Delta^{i-1} \rightarrow \Delta^{i}, j=0, \ldots, i$, are the face maps, the differential $\operatorname{Cor}\left(\Delta^{i}, X\right) \rightarrow \operatorname{Cor}\left(\Delta^{i-1}, X\right)$ is given as the composition of correspondences $x \mapsto x \circ \partial$. The following lemma is easy to check from the definitions:

Lemma 3.1. Let $f: X \rightarrow Y$ be a morphism of schemes.

a) If $X$ and $T$ are smooth and $c \in \operatorname{Cor}(T, X)$, then $\left(\operatorname{id}_{T} \times f\right)_{*} c=f \circ c$. Here the left hand side is push-forward of cycles, and the right hand side is composition of correspondences.

b) If $X$ and $Y$ are smooth and $d \in \operatorname{Cor}(Y, Z)$, then $\left(f \times \mathrm{id}_{Z}\right)^{*} d=d \circ f$. Here the left hand side is pull-back of cycles, and the right hand side is composition of correspondences.

c) If $f$ is an automorphism of the smooth scheme $X$, then $f^{*} c=f_{*}^{-1} c$ for any cycle $c$.

Let $T$ and $X$ be separated schemes of finite type over $k$ and $\sigma \in \operatorname{Gal}(\bar{k} / k)$. Then $\sigma$ acts on $\bar{X}=X \times_{k} \bar{k}$ via its action on $\bar{k}$, and on algebraic cycles by pull-back.

Lemma 3.2. The action of $\sigma$ on $\operatorname{Cor}_{\bar{k}}(\bar{T}, \bar{X})$ induced by pull-back of algebraic cycles sends a correspondence $\alpha$ to the composition $\sigma_{X}^{-1} \alpha \sigma_{T}$, where $\sigma_{X}$ and $\sigma_{T}$ are the automorphisms of $\bar{X}$ and $\bar{T}$ induced by $\sigma$. In other words, the following diagram of correspondences commutes:

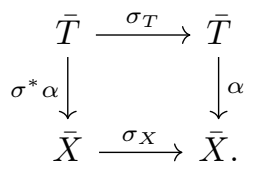

Proof. From Lemma 3.1, we have

$$
\begin{aligned}
\sigma_{X} \circ \sigma^{*} \alpha=\left(\operatorname{id}_{T} \times \sigma_{X}\right)_{*}\left(\sigma_{T}\right. & \left.\times \sigma_{X}\right)^{*} \alpha \\
& =\left(\operatorname{id}_{T} \times \sigma_{X}^{-1}\right)^{*}\left(\operatorname{id}_{T} \times \sigma_{X}\right)^{*}\left(\sigma_{T} \times \operatorname{id}_{X}\right)^{*} \alpha=\alpha \circ \sigma_{T} .
\end{aligned}
$$

Now we assume that $k=\mathbb{F}$ is a finite field. Let $\bar{X}$ be the extension to the algebraic closure $\overline{\mathbb{F}}$, and let $F$ be the Frobenius automorphism of $\overline{\mathbb{F}} / \mathbb{F}$. Let $G \cong \mathbb{Z}$ be the Weil group of $\mathbb{F}$, generated by the Frobenius $F$.

Definition 3.3. Weil-Suslin homology $H_{i}^{\mathrm{WS}}(X, A)$ with coefficients in the abelian group $A$ is defined as the homology of the cone of

$$
C_{\bullet}(\bar{X}) \otimes A \stackrel{1-F^{*}}{\longrightarrow} C \bullet(\bar{X}) \otimes A,
$$

i.e., the total complex of the double complex

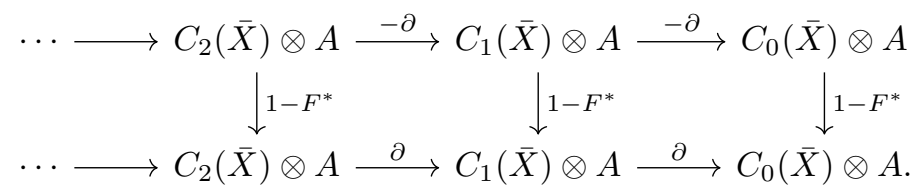


In degree $i$, the total complex consists of elements

$$
\left(x_{i}, x_{i-1}\right) \in \operatorname{Cor}\left(\bar{\Delta}^{i}, \bar{X}\right) \otimes A \oplus \operatorname{Cor}\left(\bar{\Delta}^{i-1}, \bar{X}\right) \otimes A
$$

with differential

$$
(x, y) \longmapsto\left(x \partial+y-F^{-1} y F,-y \partial\right) .
$$

The spectral sequence for double complexes gives short exact sequences

$$
0 \rightarrow H_{i}^{\mathrm{S}}(\bar{X}, A)_{G} \rightarrow H_{i}^{\mathrm{WS}}(X, A) \rightarrow H_{i-1}^{\mathrm{S}}(\bar{X}, A)^{G} \rightarrow 0
$$

where the left hand side and right hand side are the coinvariants and invariants with respect to $G$, respectively. The map $C_{\bullet}(X) \rightarrow C_{\bullet}(\bar{X})$, sending a generator $Z \subseteq X \times \Delta^{i}$ to its pull-back to the algebraic closure, has image in the kernel of $1-F^{*}$. Therefore, we obtain natural maps for $i \geq 0$.

$$
H_{i}^{\mathrm{S}}(X, A) \longrightarrow H_{i+1}^{\mathrm{WS}}(X, A) .
$$

Remark 3.4. For a torsion $\operatorname{Gal}_{\mathbb{F}}$-module $M$, we have $R \Gamma(\mathbb{F}, M) \cong\left[M \stackrel{1-F^{*}}{\longrightarrow} M\right]$, where the last complex is concentrated in (cohomological) degrees zero and one. Hence, if $A$ is a torsion group, then

$$
H_{i}^{\mathrm{WS}}(X, A)=H^{1-i}\left(R \Gamma\left(\mathbb{F}, C_{\bullet}(\bar{X}) \otimes A\right)\right) .
$$

Remark 3.5. The definition of Weil-Suslin homology depends on the finite base field $\mathbb{F}$ (via $F \in \mathrm{Gal}_{\mathbb{F}}$ ). However, if $X \rightarrow \mathbb{F}$ factors through $\mathbb{F}^{\prime} / \mathbb{F}$, then the WeilSuslin homology of $X$ does not depend on whether we consider $X$ as a scheme over $\mathbb{F}^{\prime}$ or over $\mathbb{F}$.

Proposition 3.6. An abstract blow-up diagram (4) induces a long exact sequence of Weil-Suslin homology groups

$$
\begin{aligned}
H_{1}^{\mathrm{WS}}\left(Z^{\prime}, A\right) \longrightarrow H_{1}^{\mathrm{WS}}\left(X^{\prime}, A\right) \oplus H_{1}^{\mathrm{WS}}(Z, A) \longrightarrow H_{1}^{\mathrm{WS}}(X, A) \longrightarrow \\
H_{0}^{\mathrm{WS}}\left(Z^{\prime}, A\right) \longrightarrow H_{0}^{\mathrm{WS}}\left(X^{\prime}, A\right) \oplus H_{0}^{\mathrm{WS}}(Z, A) \longrightarrow H_{0}^{\mathrm{WS}}(X, A) \longrightarrow 0 .
\end{aligned}
$$

Proof. By definition of Weil-Suslin homology, only the terms $C_{i}(\bar{?})$ for $i \leq 2$ are involved in the definition of the terms in the sequence, hence a diagram chase shows that it suffices to show that in the short exact sequence of complexes

$$
0 \rightarrow C_{\bullet}\left(\bar{Z}^{\prime}\right) \rightarrow C_{\bullet}\left(\bar{X}^{\prime}\right) \oplus C_{\bullet}(\bar{Z}) \rightarrow C_{\bullet}(\bar{X}) \rightarrow K_{\bullet} \rightarrow 0
$$

one has $H_{i}\left(K_{\bullet}\right)=0$ for $i \leq 2$. This was shown in the proof of [GS, Prop. 5.2].

Since $H_{0}^{\mathrm{S}}(\overline{\mathbb{F}}, \mathbb{Z})=\mathbb{Z}$ and $H_{i}^{\mathrm{S}}(\overline{\mathbb{F}}, \mathbb{Z})=0$ for $i \geq 1$, we can calculate the Weil-Suslin homology of the point using (15) as follows.

\section{Example 3.7.}

$$
H_{i}^{\mathrm{WS}}(\mathbb{F}, \mathbb{Z}) \cong \begin{cases}\mathbb{Z} & i=0,1 \\ 0 & i \geq 2\end{cases}
$$


Let $C$ by a smooth, proper, geometrically connected curve over $\mathbb{F}$. By $[\mathrm{Li}]$, we have

$$
H_{i}^{\mathrm{S}}(\bar{C}, \mathbb{Z}) \cong \begin{cases}\operatorname{Pic}(\bar{C}) & i=0, \\ \overline{\mathbb{F}}^{\times} & i=1, \\ 0 & i \geq 2 .\end{cases}
$$

Regarding $\overline{\mathbb{F}}_{G}^{\times}=0$ and $\operatorname{Pic}(\bar{C})_{G} \cong \mathbb{Z}$, the exact sequence (15) yields the following:

Example 3.8. Let $C$ be a smooth, proper, geometrically connected curve over $\mathbb{F}$. Then

$$
H_{i}^{\mathrm{WS}}(C, \mathbb{Z}) \cong \begin{cases}\mathbb{Z} & i=0, \\ \operatorname{Pic}(C) & i=1, \\ \mathbb{F}^{\times} & i=2, \\ 0 & i \geq 3 .\end{cases}
$$

The following improves Ge3, Prop. 7.8]:

Proposition 3.9. Let $X$ be a connected, separated scheme of finite type over $\mathbb{F}$. Then the structure map induces an isomorphism

$$
\operatorname{deg}: H_{0}^{\mathrm{WS}}(X, \mathbb{Z}) \stackrel{\sim}{\longrightarrow} H_{0}^{\mathrm{WS}}(\mathbb{F}, \mathbb{Z}) \cong \mathbb{Z} .
$$

Proof. We have $H_{0}^{\mathrm{WS}}(X, \mathbb{Z}) \cong H_{0}^{\mathrm{S}}(\bar{X}, \mathbb{Z})_{G}$, hence deg is surjective and it remains to show that its kernel is trivial. Since elements in $H_{0}^{\mathrm{S}}(\bar{X}, \mathbb{Z})$ are represented by zero-cycles, any element of $H_{0}^{\mathrm{WS}}(X, \mathbb{Z})$ comes by push-forward from $H_{0}^{\mathrm{WS}}(C, \mathbb{Z})$ for some connected curve $C \subset X$ (use, e.g., $\mathrm{Mu}$, II $\S 6$ Lemma). We therefore can assume that $X=C$ is a connected curve.

If $C^{\prime} \rightarrow C$ is finite and surjective, then $H_{0}^{\mathrm{WS}}\left(C^{\prime}, \mathbb{Z}\right) \rightarrow H_{0}^{\mathrm{WS}}(C, \mathbb{Z})$ is surjective. Moreover, any element of degree zero in $H_{0}^{\mathrm{WS}}(\bar{C}, \mathbb{Z})$ can be lifted to an element in the kernel of the multi-degree map

$$
H_{0}^{\mathrm{WS}}\left(C^{\prime}, \mathbb{Z}\right) \longrightarrow \mathbb{Z}^{\pi_{0}\left(C^{\prime}\right)} .
$$

We therefore can assume that $C$ is a normal, connected curve. Moreover, we can assume that $C$ has an $\mathbb{F}$-rational point (use Remark 3.5). Let $\mathcal{A}$ be the semi-abelian Albanese variety of $C$. Then, by [SV1, Thm. 3.1] (and [Li] if $C$ is proper), the degree zero part of $H_{0}^{\mathrm{S}}(\bar{C}, \mathbb{Z})$ is isomorphic to $\mathcal{A}(\overline{\mathbb{F}})$. The $G$-coinvariants of this group are both finite and divisible, hence trivial.

Corollary 3.10. The canonical injection

$$
H_{1}^{\mathrm{WS}}(X, \mathbb{Z}) / m \longrightarrow H_{1}^{\mathrm{WS}}(X, \mathbb{Z} / m)
$$

is an isomorphism for any $m \geq 1$.

Proof. The cokernel is isomorphic to $\operatorname{ker}\left(H_{0}^{\mathrm{WS}}(X, \mathbb{Z}) \stackrel{m}{\rightarrow} H_{0}^{\mathrm{WS}}(X, \mathbb{Z})\right)=0$.

\section{Duality}

We say that "resolution of singularities holds for schemes of dimension $\leq d$ over a perfect field $k$ " if the following two conditions are satisfied.

(1) For any integral separated scheme of finite type $X$ of dimension $\leq d$ over $k$, there exists a projective birational morphism $Y \rightarrow X$ with $Y$ smooth over $k$ which is an isomorphism over the regular locus of $X$. 
(2) For any integral smooth scheme $X$ of dimension $\leq d$ over $k$ and any birational proper morphism $Y \rightarrow X$ there exists a tower of morphisms $X_{n} \rightarrow X_{n-1} \rightarrow$ $\cdots \rightarrow X_{0}=X$, such that $X_{n} \rightarrow X_{n-1}$ is a blow-up with a smooth center for $i=1, \ldots, n$, and such that the composite morphism $X_{n} \rightarrow X$ factors through $Y \rightarrow X$.

In this paper, we do not use the full duality statement below, but only the equality of the orders of the respective groups.

Theorem 4.1. Let $X$ be separated and of finite type over the finite field $\mathbb{F}$ of characteristic $p$. If $m$ is prime to $p$, then there is a perfect pairing of finite groups

$$
H_{i}^{\mathrm{WS}}(X, \mathbb{Z} / m) \times H_{\mathrm{et}}^{i}(X, \mathbb{Z} / m) \longrightarrow \mathbb{Z} / m .
$$

If $X$ is smooth and resolution of singularities for schemes of dimension $\leq \operatorname{dim} X+1$ holds over $\mathbb{F}$, then there is a perfect pairing of finite groups for any $r \geq 1$,

$$
H_{1}^{\mathrm{WS}}\left(X, \mathbb{Z} / p^{r}\right) \times H_{t}^{1}\left(X, \mathbb{Z} / p^{r}\right) \longrightarrow \mathbb{Z} / p^{r}
$$

Proof. By Ge3, Thm. 5.4, Thm.5.5], we have a perfect pairing of finite groups

$$
H_{i-1}^{\mathrm{GS}}(X, \mathbb{Z} / m) \times H_{\mathrm{et}}^{i}(X, \mathbb{Z} / m) \longrightarrow \mathbb{Z} / m
$$

where $H_{i-1}^{\mathrm{GS}}$ is Galois-Suslin homology. By [Ge3, $\left.\S 7.1\right]$, we have $H_{i-1}^{\mathrm{GS}}(X, \mathbb{Z} / m) \cong$ $H_{i}^{\mathrm{WS}}(X, \mathbb{Z} / m)$, showing the first statement note that $H_{i}^{\mathrm{WS}}$ is denoted by $H_{i}^{a r}$ in Ge3]).

For the second statement, let $X^{\prime}$ be a smooth, proper variety containing $X$ as a dense open subscheme. Then, by [GS, Prop.6.2], we have $H_{i}^{\mathrm{S}}\left(\bar{X}, \mathbb{Z} / p^{r}\right) \cong$ $H_{i}^{\mathrm{S}}\left(\bar{X}^{\prime}, \mathbb{Z} / p^{r}\right)$ for $i=0,1$, and the exact sequence (15) above implies that

$$
H_{1}^{\mathrm{WS}}\left(X, \mathbb{Z} / p^{r}\right) \cong H_{1}^{\mathrm{WS}}\left(X^{\prime}, \mathbb{Z} / p^{r}\right)
$$

Furthermore, $H_{t}^{1}\left(X, \mathbb{Z} / p^{r}\right) \cong H_{\text {et }}^{1}\left(X^{\prime}, \mathbb{Z} / p^{r}\right)$ by [GS, Prop. 2.10]. Hence we may assume that $X=X^{\prime}$ is smooth and proper.

Let $\mathbb{Z}_{X}^{c}$ be the complex of étale sheaves on $X$ which associates to $U \rightarrow X$ the Bloch complex $z_{0}(U, \bullet)$. Then, for smooth proper $X, H_{\mathrm{et}}^{i}\left(X, \mathbb{Z} / p^{r}\right)$ is dual to $\operatorname{Ext}_{X}^{2-i}\left(\mathbb{Z} / p^{r}, \mathbb{Z}_{X}^{c}\right)$ by [Ge4, Thm. 5.1]. Furthermore,

$$
\begin{array}{rlr}
\operatorname{Ext}_{X}^{2-i}\left(\mathbb{Z} / p^{r}, \mathbb{Z}_{X}^{c}\right) & \cong \operatorname{Ext}_{X, \mathbb{Z} / p^{r}}^{1-i}\left(\mathbb{Z} / p^{r}, \mathbb{Z}_{X}^{c} / p^{r}\right) \quad \text { (by [Ge4, Lem. 2.4]) } \\
& \cong H_{\mathrm{et}}^{1-i}\left(X, \mathbb{Z}_{X}^{c} / p^{r}\right) \\
& \cong H^{1-i}\left(R \Gamma\left(\mathbb{F}, R \Gamma\left(\bar{X}_{\mathrm{et}}, \mathbb{Z}_{\bar{X}}^{c} / p^{r}\right)\right)\right) \\
& \cong H^{1-i}\left(R \Gamma\left(\mathbb{F}, \mathbb{Z}_{\bar{X}}^{c} / p^{r}(\bar{X})\right)\right) \quad \text { (by [Ge4, Thm. 3.1]). }
\end{array}
$$

The natural map

$$
C \bullet(\bar{X}) \otimes \mathbb{Z} / p^{r} \longrightarrow \mathbb{Z}_{\bar{X}}^{c} / p^{r}(\bar{X})
$$

induces an isomorphism on $H^{j}$ for $j=-1,0$ by [SS, Thm. 2.7] (the assumption $\operatorname{dim} X \leq 2$ is unnecessary and not used in the proof). Hence for $i=1$ we obtain

$$
\begin{aligned}
H^{0}\left(R \Gamma\left(\mathbb{F}, \mathbb{Z}_{\bar{X}}^{c} / p^{r}(\bar{X})\right)\right) & \cong H^{0}\left(R \Gamma\left(\mathbb{F}, C \bullet(\bar{X}) \otimes \mathbb{Z} / p^{r}\right)\right) \\
& \cong H_{1}^{\mathrm{WS}}\left(X, \mathbb{Z} / p^{r}\right) \quad \text { (by Remark 3.4) } .
\end{aligned}
$$

This concludes the proof. 


\section{The reciprocity map}

For any $X$ and any abelian group $A$, we construct a functorial pairing

$$
H_{1}^{\mathrm{WS}}(X, \mathbb{Z}) \times H_{\mathrm{W}, t}^{1}(X, A) \stackrel{\lfloor,\rangle}{\longrightarrow} A,
$$

which induces natural maps

$$
\operatorname{Hom}\left(\Pi_{1}^{t, \mathrm{ab}}(X)_{\mathrm{W}}, A\right) \cong H_{\mathrm{W}, t}^{1}(X, A) \longrightarrow \operatorname{Hom}\left(H_{1}^{\mathrm{WS}}(X, \mathbb{Z}), A\right)
$$

for any abelian (pro-)group $A$, hence the Yoneda lemma induces

$$
\operatorname{rec}_{X}: H_{1}^{\mathrm{ws}}(X, \mathbb{Z}) \longrightarrow \Pi_{1}^{t, \mathrm{ab}}(X)_{\mathrm{W}} .
$$

The pairing (17) should satisfy two conditions. First of all, the composite of $\operatorname{rec}_{X}$ with the natural map $H_{0}^{\mathrm{S}}(X, \mathbb{Z}) \rightarrow H_{1}^{\mathrm{WS}}(X, \mathbb{Z})$ should be the map

$$
r_{X}: H_{0}^{\mathrm{S}}(X, \mathbb{Z}) \longrightarrow \Pi_{1}^{t, \mathrm{ab}}(X)_{\mathrm{W}}
$$

which sends the class $[x]$ of a closed point $x \in X$ to its Frobenius automorphism in $\Pi_{1}^{t, \mathrm{ab}}(X)_{\mathrm{w}}$.

Secondly, we want (17) to be compatible with the pairing over $\overline{\mathbb{F}}$ considered in GS: in loc. cit. we considered (over any algebraically closed field) a natural pairing

$$
H_{1}^{\mathrm{S}}(\bar{X}, \mathbb{Z}) \times H_{t}^{1}(\bar{X}, A) \longrightarrow A,
$$

which is defined by pulling back torsors along finite correspondences. For $A=\mathbb{Z} / m$, $(m, \operatorname{char}(\overline{\mathbb{F}}))=1$, the induced homomorphism

$$
\operatorname{Hom}\left(H_{1}^{\mathrm{S}}(\bar{X}, \mathbb{Z}), \mathbb{Z} / m\right) \longrightarrow H_{\mathrm{et}}^{1}(\bar{X}, \mathbb{Z} / m)
$$

coincides with the composite of $\operatorname{Hom}\left(H_{1}^{\mathrm{S}}(\bar{X}, \mathbb{Z}), \mathbb{Z} / m\right) \stackrel{\text { can }}{\hookrightarrow} H_{\mathrm{S}}^{1}(\bar{X}, \mathbb{Z} / m)$ with the Suslin-Voevodsky comparison isomorphism ([SV1, Cor. 7.8])

$$
H_{\mathrm{S}}^{1}(\bar{X}, \mathbb{Z} / m) \stackrel{S V}{\sim} H_{\mathrm{et}}^{1}(\bar{X}, \mathbb{Z} / m) .
$$

We want to construct the pairing (17) in such a way that the diagram

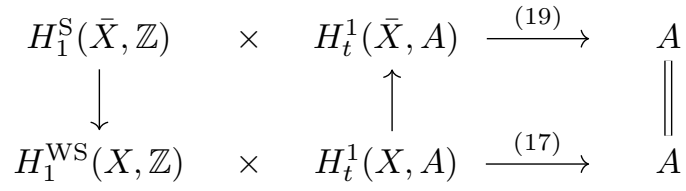

commutes. In [GS, §4] an explicit interpretation of (19) in terms of qfh-sheaves is given. This motivates the following construction of the pairing (17).

Let $A$ be an abelian group and $A \rightarrow \mathrm{I}^{\bullet}$ an injective resolution of the constant sheaf $A$ in the category of qfh-sheaves. An element of $H_{\mathrm{W}, t}^{1}(X, A)$ is represented by a pair

$$
(\alpha, \beta) \in \mathrm{I}^{1}(\bar{X}) \oplus \mathrm{I}^{0}(\bar{X}),
$$

with $d \alpha=0,[\alpha] \in H_{t}^{1}(\bar{X}, A)$ and $d \beta=\alpha-F^{*} \alpha$. An element in $H_{1}^{\mathrm{WS}}(X, \mathbb{Z})$ is represented by a pair

$$
(x, y) \in \operatorname{Cor}\left(\bar{\Delta}^{1}, \bar{X}\right) \oplus \operatorname{Cor}\left(\bar{\Delta}^{0}, \bar{X}\right),
$$

with $x \partial=F^{-1} y F-y$. 
Since $H_{t}^{1}\left(\bar{\Delta}^{1}, A\right)=0=H_{\mathrm{et}}^{1}\left(\bar{\Delta}^{0}, A\right)$, we can find $s \in \mathrm{I}^{0}\left(\bar{\Delta}^{1}\right)$ with $d s=x^{*} F^{*} \alpha \in$ $\mathrm{I}^{1}\left(\bar{\Delta}^{1}\right)$ and $t \in \mathrm{I}^{0}\left(\bar{\Delta}^{0}\right)$ with $d t=y^{*} \alpha \in \mathrm{I}^{1}\left(\bar{\Delta}^{0}\right)$. Then

$$
\langle(x, y),(\alpha, \beta)\rangle:=F^{*} t-t-\partial^{*} s+y^{*} \beta
$$

lies in

$$
A=H_{\mathrm{et}}^{0}\left(\bar{\Delta}^{0}, A\right)=\operatorname{ker}\left(\mathrm{I}^{0}\left(\bar{\Delta}^{0}\right) \stackrel{d}{\longrightarrow} \mathrm{I}^{1}\left(\bar{\Delta}^{0}\right)\right) .
$$

Indeed, we have

$$
\begin{aligned}
d\left(F^{*} t-t-\partial^{*} s+y^{*} \beta\right)=F^{*} & y^{*} \alpha-y^{*} \alpha-\partial^{*} x^{*} F^{*} \alpha+y^{*}\left(\alpha-F^{*} \alpha\right) \\
& =F^{*} y^{*} \alpha-\left(F^{*} y^{*} F^{*-1}-y^{*}\right)\left(F^{*} \alpha\right)-y^{*} F^{*} \alpha=0 .
\end{aligned}
$$

One checks without difficulty that $\langle(x, y),(\alpha, \beta)\rangle$ does not depend on the choices of $s$ and $t$.

Lemma 5.1. $\langle(x, y),(\alpha, \beta)\rangle \in A$ only depends on the class of $(x, y)$ in $H_{1}^{\mathrm{WS}}(X, \mathbb{Z})$ and on the class of $(\alpha, \beta)$ in $H_{\mathrm{W}, t}^{1}(X, A)$.

Proof. For $\gamma \in \mathrm{I}^{0}(\bar{X}), s=x^{*} F^{*} \gamma$ and $t=y^{*} \gamma$ satisfy the condition and we have

$$
\begin{aligned}
& \left\langle(x, y),\left(d \gamma, \gamma-F^{*} \gamma\right)\right\rangle=F^{*} t-t-\partial^{*} s+y^{*}\left(\gamma-F^{*} \gamma\right) \\
& \quad=F^{*} y^{*} \gamma-y^{*} \gamma-(x \delta)^{*} F^{*} \gamma=F^{*} y^{*} \gamma-y^{*} \gamma-F^{*} y^{*} \gamma+y^{*} F^{*} \gamma=0 .
\end{aligned}
$$

Let $(u, v) \in \operatorname{Cor}\left(\bar{\Delta}^{2}, \bar{X}\right) \oplus \operatorname{Cor}\left(\bar{\Delta}^{1}, \bar{X}\right)$. Since $H_{t}^{1}\left(\bar{\Delta}^{2}, A\right)=0=H_{t}^{1}\left(\bar{\Delta}^{1}, A\right)$, we can find $\sigma \in \mathrm{I}^{0}\left(\bar{\Delta}^{2}\right)$ with $d \sigma=u^{*} F^{*} \alpha$ and $\tau \in \mathrm{I}^{0}\left(\bar{\Delta}^{1}\right)$ with $d \tau=-v^{*} \alpha$. Then $s=\partial^{*} \sigma+F^{*} \tau-\tau-v^{*} \beta$ and $t=\partial^{*} \tau$ satisfy

$$
d s=\left(u \partial+v-F^{-1} v F\right) F^{*} \alpha, \quad d t=(-v \partial)^{*} \alpha
$$

and

$$
\begin{aligned}
& \left\langle\left(u \partial+v-F^{-1} v F,-v \partial\right),(\alpha, \beta)\right\rangle=F^{*} t-t-\partial^{*} s-(v \partial)^{*} \beta= \\
& F^{*} \partial^{*} \tau-\partial^{*} \tau-\partial^{*}\left(\partial+\sigma^{*} F^{*} \tau-\tau-v^{*} \beta\right)-\partial^{*} v^{*} \beta=0 .
\end{aligned}
$$

By Lemma 5.1, we obtain the pairing (17) and the reciprocity map (18).

Lemma 5.2. If $f: X \rightarrow Y$ is an $\mathbb{F}$-morphism, then the diagram

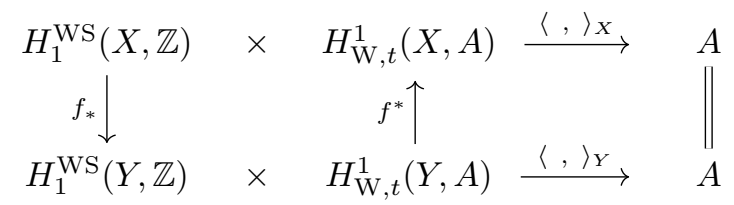

commutes, hence $\operatorname{rec}_{X}: H_{1}^{\mathrm{WS}}(X, \mathbb{Z}) \rightarrow \Pi_{1}^{t, \mathrm{ab}}(X)_{\mathrm{W}}$ is functorial in $X$.

Proof. Let $A \rightarrow \mathrm{I}^{\bullet}$ an injective resolution of the constant sheaf $A$ in the category of qfh-sheaves. Let $c \in H_{\mathrm{W}, t}^{1}(Y, A)$ be represented by

$$
(\alpha, \beta) \in \mathrm{I}^{1}(\bar{Y}) \oplus \mathrm{I}^{0}(\bar{Y}),
$$

with $d \alpha=0,[\alpha] \in H_{t}^{1}(\bar{Y}, A)$ and $d \beta=\alpha-F^{*} \alpha$. Furthermore, let $\zeta \in H_{1}^{\mathrm{WS}}(X, \mathbb{Z})$ be represented by

$$
(x, y) \in \operatorname{Cor}\left(\bar{\Delta}^{1}, \bar{X}\right) \oplus \operatorname{Cor}\left(\bar{\Delta}^{0}, \bar{X}\right),
$$


with $x \partial=F^{-1} y F-y$. We have to show that $\left\langle\zeta, f^{*}(c)\right\rangle_{X}=\left\langle f_{*}(\zeta), c\right\rangle_{Y}$. This follows directly from the construction: First note that $f_{*}(\zeta)$ is represented by $(f \circ x, f \circ y)$. Choose $s \in \mathrm{I}^{0}\left(\bar{\Delta}^{1}\right)$ with

$$
d s=(f \circ x)^{*} F^{*} \alpha \in \mathrm{I}^{1}\left(\bar{\Delta}^{1}\right)
$$

and $t \in \mathrm{I}^{0}\left(\bar{\Delta}^{0}\right)$ with $d t=(f \circ y)^{*} \alpha \in \mathrm{I}^{1}\left(\bar{\Delta}^{0}\right)$. Then

$$
\langle(f \circ x, f \circ y),(\alpha, \beta)\rangle_{Y}=F^{*} t-t-\partial^{*} s+(f \circ y)^{*} \beta
$$

by definition. Since $d s=x^{*} F^{*}\left(f^{*}(\alpha)\right), d t=y^{*}\left(f^{*}(\alpha)\right)$, we obtain

$$
\begin{aligned}
\left\langle(x, y), f^{*}(\alpha, \beta)\right\rangle_{X} & =F^{*} t-t-\partial^{*} s+y^{*}\left(f^{*}(\beta)\right) \\
& =F^{*} t-t-\partial^{*} s+(f \circ y)^{*} \beta \\
& =\langle(f \circ x, f \circ y),(\alpha, \beta)\rangle_{Y},
\end{aligned}
$$

showing the assertion.

Proposition 5.3. The composite of $\operatorname{rec}_{X}$ with the natural map $H_{0}^{\mathrm{S}}(X, \mathbb{Z}) \rightarrow$ $H_{1}^{\mathrm{WS}}(X, \mathbb{Z})$ is the map

$$
r_{X}: H_{0}^{\mathrm{S}}(X, \mathbb{Z}) \longrightarrow \Pi_{1}^{t, \mathrm{ab}}(X)_{\mathrm{W}}
$$

which sends the class $[x]$ of a closed point $x \in X$ to its Frobenius automorphism in $\Pi_{1}^{t, \mathrm{ab}}(X)_{\mathrm{W}}$.

Proof. By functoriality, it suffices to consider the case $X=\Delta^{0}$. In this case, we have natural identifications $\mathbb{Z}=H_{0}^{\mathrm{S}}\left(\Delta^{0}, \mathbb{Z}\right)=H_{1}^{\mathrm{WS}}\left(\Delta^{0}, \mathbb{Z}\right)$ (sending $1 \in \mathbb{Z}$ to $\left.\operatorname{id}_{\Delta^{0}} \in \operatorname{Cor}\left(\Delta^{0}, \Delta^{0}\right)\right)$, and for any abelian group $A$, we have $A=H_{\text {et }}^{0}\left(\bar{\Delta}^{0}, A\right)_{G}=$ $H_{\mathrm{W}, t}^{1}\left(\Delta^{0}, A\right)$. With respect to these identifications, the pairing (17) is just multiplication $\mathbb{Z} \times A \rightarrow A,(n, a) \mapsto n a$. Furthermore, the isomorphism of Proposition 2.4

$$
A=H_{\mathrm{W}, t}^{1}\left(\Delta^{0}, A\right) \stackrel{\sim}{\longrightarrow} \operatorname{Hom}\left(\Pi_{1}^{t, \mathrm{ab}}\left(\Delta^{0}\right)_{\mathrm{W}}, A\right)=\operatorname{Hom}(G, A)
$$

maps $a \in A$ to the homomorphism $G \rightarrow A$, which sends the Frobenius $F \in G \cong \mathbb{Z}$ to $a$. Using all this, the statement of the proposition follows from the definition of the reciprocity map.

\section{Comparison of blow-up sequences}

If $A=\mathbb{Z} / m$, then Weil-etale and etale cohomology agree, and by Corollary 3.10 , the pairing (17) induces a pairing

$$
H_{1}^{\mathrm{WS}}(X, \mathbb{Z} / m) \times H_{t}^{1}(X, \mathbb{Z} / m) \longrightarrow \mathbb{Z} / m,
$$

and hence a map

$$
\Phi_{X}^{1}: H_{1}^{\mathrm{WS}}(X, \mathbb{Z} / m) \longrightarrow H_{t}^{1}(X, \mathbb{Z} / m)^{*},
$$

which is the $\bmod m$-version of $\operatorname{rec}_{X}$.

In addition, we consider the pairing

$$
H_{0}^{\mathrm{WS}}(X, \mathbb{Z} / m) \times H_{\mathrm{et}}^{0}(X, \mathbb{Z} / m) \longrightarrow \mathbb{Z} / m
$$

defined as follows: Choose $x \in \operatorname{Cor}\left(\bar{\Delta}^{0}, \bar{X}\right)$ representing a class in $H_{0}^{\mathrm{WS}}(X, \mathbb{Z} / m)$ $=H_{0}^{\mathrm{S}}(\bar{X}, \mathbb{Z} / m)_{G}$ and $\alpha \in I^{0}(\bar{X})$ with $d \alpha=0$ and $\alpha-F^{*} \alpha=0$. Then put $\langle x, \alpha\rangle=x^{*} \alpha \in H_{\text {et }}^{0}\left(\bar{\Delta}^{0}, \mathbb{Z} / m\right)=\mathbb{Z} / m$. We obtain a map

$$
\Phi_{X}^{0}: H_{0}^{\mathrm{WS}}(X, \mathbb{Z} / m) \longrightarrow H_{\mathrm{et}}^{0}(X, \mathbb{Z} / m)^{*} .
$$

The maps $\Phi^{0}$ and $\Phi^{1}$ extend in a natural way to non-connected schemes. They induce a map from the exact sequence of Proposition 3.6 to the dual of the exact sequence of Proposition 2.1. The compatibility with the boundary map is given by 
Proposition 6.1. For any abstract blow-up square

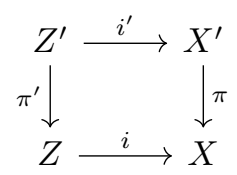

the following diagram is commutative

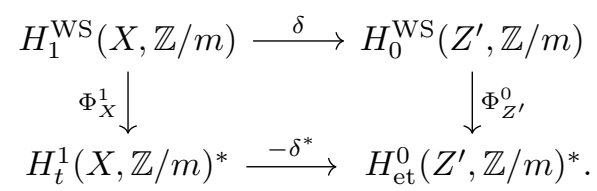

Here $\delta$ is the boundary map of the exact sequence of Proposition [3.6 and $\delta^{*}$ is the dual of the boundary map of the exact sequence of Proposition [2.1.

Proof. We have to show that the diagram

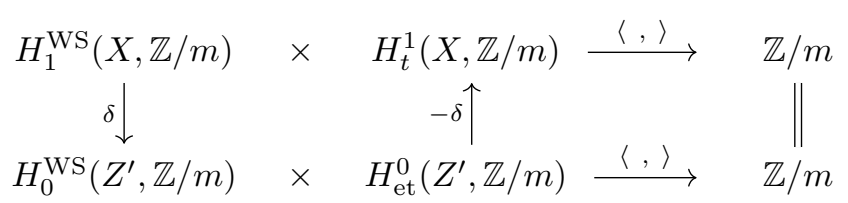

commutes. Let $a \in H_{1}^{\mathrm{WS}}(X, \mathbb{Z} / m)$ and $b \in H_{\mathrm{et}}^{0}\left(Z^{\prime}, \mathbb{Z} / m\right)$. We put

$$
C_{i}^{\mathrm{W}}(X)=C_{i}(\bar{X}) \otimes \mathbb{Z} / m \oplus C_{i-1}(\bar{X}) \otimes \mathbb{Z} / m,
$$

i.e., $C_{\bullet}^{\mathrm{W}}(X)$ is the complex calculating $H_{\bullet}^{\mathrm{WS}}(X, \mathbb{Z} / m)$. Consider the diagram

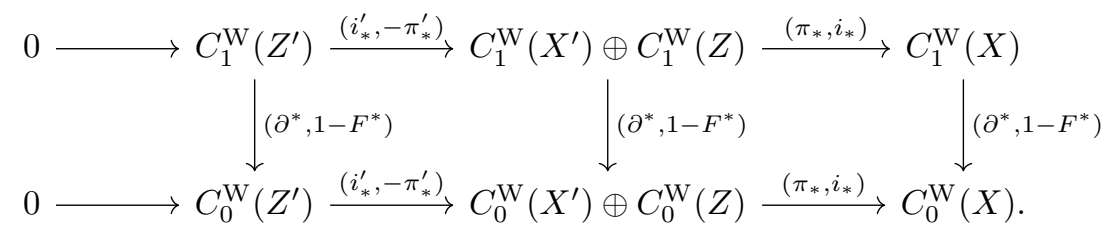

By Proposition 3.6 and its proof, $a \in H_{1}^{\mathrm{WS}}(X, \mathbb{Z} / m)$ can be represented by a cocycle $\alpha \in C_{1}^{\mathrm{W}}(X)$ which can be lifted to $C_{1}^{\mathrm{W}}\left(X^{\prime}\right) \oplus C_{1}^{\mathrm{W}}(Z)$. We choose $\hat{\alpha} \in C_{1}^{\mathrm{W}}\left(X^{\prime}\right) \oplus$ $C_{1}^{\mathrm{W}}(Z)$ with $\left(\pi_{*}, i_{*}\right)(\hat{\alpha})=\alpha$, hence $\left(\pi_{*}, i_{*}\right)\left(\partial^{*}, 1-F^{*}\right)(\hat{\alpha})=0$. We conclude that $\delta(a) \in H_{0}^{\mathrm{WS}}\left(Z^{\prime}, \mathbb{Z} / m\right)$ is represented by an element $\gamma \in C_{0}^{\mathrm{W}}\left(Z^{\prime}\right)$ with

$$
\left(i_{*}^{\prime},-\pi_{*}^{\prime}\right)(\gamma)=\left(\partial^{*}, 1-F^{*}\right)(\hat{\alpha})
$$

Let $\mathrm{I}^{\bullet}$ be an injective resolution of $\mathbb{Z} / m$ in the category of sheaves of $\mathbb{Z} / m$-modules on the $h$-site on $\mathrm{Sch} / \mathbb{F}$, and let $\beta \in \mathrm{I}^{0}\left(Z^{\prime}\right), d \beta=0$, be a representative of $b \in$ $H_{\mathrm{et}}^{0}\left(Z^{\prime}, \mathbb{Z} / m\right)$. Consider the diagram

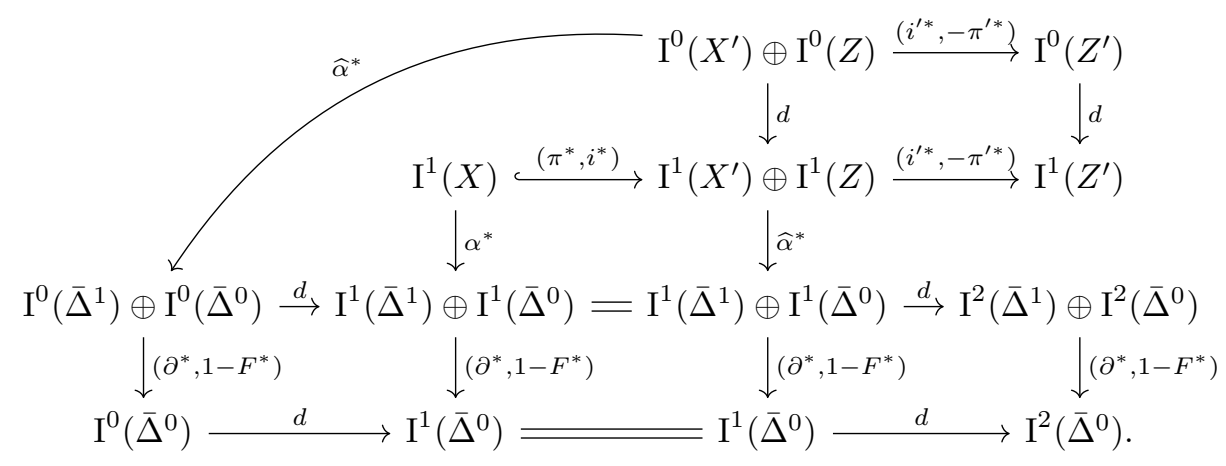


Since the complex coker $\left(\mathrm{I}^{\bullet}\left(X^{\prime}\right) \oplus \mathrm{I}^{\bullet}(Z) \rightarrow \mathrm{I}^{\bullet}\left(Z^{\prime}\right)\right)$ is exact (cf. the exact triangle (6) in the proof of Proposition 2.1), we find $\hat{\beta} \in \mathrm{I}^{0}\left(X^{\prime}\right) \oplus \mathrm{I}^{0}(Z)$ with $\left(i^{\prime *},-{\pi^{\prime *}}^{\prime *}(\hat{\beta})=\beta\right.$. By the argument of [MVW, Lem. 12.7], the sequence

$$
0 \rightarrow \mathcal{F}(X) \rightarrow \mathcal{F}\left(X^{\prime}\right) \oplus \mathcal{F}(Z) \rightarrow \mathcal{F}\left(Z^{\prime}\right)
$$

is exact for every $h$-sheaf $\mathcal{F}$. Therefore the second line in the diagram is exact and there is a unique $\varepsilon \in \mathrm{I}^{1}(X)$ with $\left(\pi^{*}, i^{*}\right)(\varepsilon)=d \hat{\beta}$ representing $\delta(b) \in H_{t}^{1}(X, \mathbb{Z} / m)$. From $\hat{\alpha}^{*}(d \hat{\beta})=\alpha^{*}(d \varepsilon)$ it follows that

$$
d\left(\hat{\alpha}^{*}(\hat{\beta})\right)=\alpha^{*}(\varepsilon) \in \operatorname{ker}\left(\partial^{*}, 1-F^{*}\right) .
$$

By definition, we have

$$
\langle a, \delta b\rangle=-\left(\partial^{*}, 1-F^{*}\right) \hat{\alpha}^{*}(\hat{\beta}) \in \operatorname{ker}\left(\mathrm{I}^{0}\left(\bar{\Delta}^{0}\right) \rightarrow \mathrm{I}^{1}\left(\bar{\Delta}^{0}\right)\right)=H_{\mathrm{et}}^{0}\left(\bar{\Delta}^{0}, \mathbb{Z} / m\right) .
$$

On the other hand, $\langle\delta a, b\rangle=\gamma^{*}(b) \in H_{\mathrm{et}}^{0}\left(\bar{\Delta}^{0}, \mathbb{Z} / m\right)$ is represented by $\gamma^{*} \beta \in \mathrm{I}^{0}\left(\Delta^{0}\right)$ and

$$
\gamma^{*} \beta=\gamma^{*}\left(i^{*},-\pi^{\prime *}\right)(\hat{\beta})=\left(i_{*}^{\prime}(\gamma),-\pi_{*}^{\prime}(\gamma)\right)^{*}(\hat{\beta})=\left(\left(\partial^{*}, 1-F^{*}\right)(\hat{\alpha})\right)^{*}(\hat{\beta}) .
$$

Now we write $\hat{\alpha}=\left(\hat{\alpha}_{1}, \hat{\alpha}_{2}\right)$ with $\hat{\alpha}_{1} \in C_{1}\left(\bar{X}^{\prime}\right) \oplus C_{1}(\bar{Z})$ and $\hat{\alpha}_{2} \in C_{0}\left(\bar{X}^{\prime}\right) \oplus C_{0}(\bar{Z})$.

Then $\left(\partial^{*}, 1-F^{*}\right)(\hat{\alpha})=\alpha_{1} \partial+\alpha_{2}-F^{-1} \alpha_{2} F$. Since $F^{*}(\hat{\beta})=\hat{\beta}$, we conclude

$$
\left(\left(\partial^{*}, 1-F^{*}\right)(\hat{\alpha})\right)^{*}(\hat{\beta})=\left(\delta^{*}, 1-F^{*}\right) \hat{\alpha}^{*}(\hat{\beta}) .
$$

This completes the proof.

\section{Proof of the main theorem}

To prove our main theorem, we first consider finite coefficients.

Proposition 7.1. The map $\Phi_{X}^{0}$ is an isomorphism for any $X$ and $m$.

Proof. We can assume that $X$ is connected. Then, by Proposition 3.9, the degree map induces an isomorphism $H_{0}^{\mathrm{WS}}(X, \mathbb{Z} / m) \stackrel{\sim}{\rightarrow} H_{0}^{\mathrm{WS}}(\mathbb{F}, \mathbb{Z} / m) \cong \mathbb{Z} / m$. Furthermore, $H_{\mathrm{et}}^{0}(\mathbb{F}, \mathbb{Z} / m) \stackrel{\sim}{\rightarrow} H_{\mathrm{et}}^{0}(X, \mathbb{Z} / m)$. Hence, by functoriality, we can reduce the statement to the case $X=\operatorname{Spec}(\mathbb{F})$, where it is easy.

Theorem 7.2. For any separated scheme of finite type over a finite field $\mathbb{F}$, the map

$$
\Phi_{X}^{1}: H_{1}^{\mathrm{WS}}(X, \mathbb{Z} / m) \longrightarrow H_{t}^{1}(X, \mathbb{Z} / m)^{*}
$$

is surjective. It is an isomorphism if $m$ is prime to the characteristic or if resolutions of singularities holds for schemes of dimension $\leq \operatorname{dim} X+1$ over $\mathbb{F}$.

Proof. By Propositions 2.1, 3.6 and 6.1, and induction on the dimension, we can assume that $X$ is normal and connected. Then, by Proposition 5.3 and ChebotarevLang density, the composite

$$
H_{0}^{\mathrm{S}}(X, \mathbb{Z}) / m \longrightarrow H_{1}^{\mathrm{WS}}(X, \mathbb{Z} / m) \stackrel{\Phi_{X}^{1}}{\longrightarrow} H_{t}^{1}(X, \mathbb{Z} / m)^{*}
$$

is surjective, hence so is $\Phi_{X}^{1}$. To get the isomorphism, we note that by Theorem 4.1, the source and the target of $\Phi_{X}^{1}$ have the same order under the given hypothesis. 
Proof of Theorem 1.2. Consider the diagram (for any $m$ )

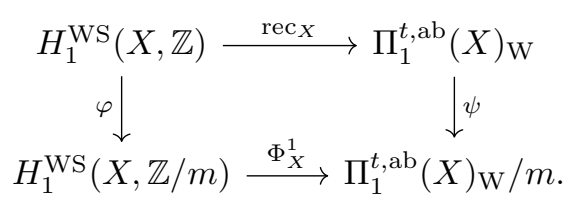

The composite $\Phi_{X}^{1} \circ \varphi$ is surjective by Corollary 3.10 and Theorem 7.2 . Hence the cokernel of $\operatorname{rec}_{X}$ is divisible. Since $\Pi_{1}^{t, \mathrm{ab}}(X)_{\mathrm{W}}$ is finitely generated, all divisible elements of $H_{1}^{\mathrm{WS}}(X, \mathbb{Z})$ are in the kernel of $\operatorname{rec}_{X}$, and the cokernel of rec ${ }_{X}$ is finitely generated and divisible, hence trivial.

Now assume that resolution of singularities holds for schemes of dimension $\leq$ $\operatorname{dim} X+1$ over $\mathbb{F}$. Then $\Phi_{X}^{1}$ is an isomorphism for all $m$. Hence the kernel of $\operatorname{rec}_{X}$ is the set div $H_{1}^{\mathrm{WS}}(X, \mathbb{Z})$ of divisible elements. This agrees with the maximal divisible subgroup by the following Lemma 7.3 .

Lemma 7.3. Let $A$ be an abelian group. If $A / \operatorname{div} A$ is finitely generated, then $\operatorname{div} A$ is divisible.

Proof. Let $B=A / \operatorname{div} A$, choose an integer $n$ such that $n B$ is free, and let $C \subseteq A$ be the inverse image of $n B$ in $A$. Then $\operatorname{div} C=\operatorname{div} A$ because $n A \subseteq C \subseteq A$. By freeness of $n B$, we obtain that $C=n B \oplus \operatorname{div} A$, hence $\operatorname{div} A=\operatorname{div} C=\operatorname{div} \operatorname{div} A$.

\section{The case of proper curves}

In this section we illustrate the results of this paper in the case of proper curves.

Let $C$ be a connected, proper curve over $\mathbb{F}$. The morphism $C_{\text {red }} \rightarrow C$ induces an isomorphism on Weil-Suslin homology as well as on fundamental groups. We therefore may assume that $C$ is reduced. We first note that

$$
H_{1}^{\mathrm{WS}}(C, \mathbb{Z})
$$

is a finitely generated abelian group. This follows by applying Proposition 3.6 to the normalization morphism $\widetilde{C} \rightarrow C$ and by using Examples 3.7 and 3.8 Furthermore, resolution of singularities holds for schemes of dimension $\leq 2$ over $\mathbb{F}$. Hence Theorem 1.2 yields the reciprocity isomorphism

$$
\operatorname{rec}_{C}: H_{1}^{\mathrm{WS}}(C, \mathbb{Z}) \stackrel{\sim}{\longrightarrow} \Pi_{1}^{\mathrm{ab}}(C)_{\mathrm{W}} .
$$

Since $C$ is proper, we have $\mathrm{CH}_{0}(C)=H_{0}^{\mathrm{S}}(C, \mathbb{Z})$, hence (16) yields an injection

$$
\phi: \mathrm{CH}_{0}(C) \hookrightarrow H_{1}^{\mathrm{WS}}(C, \mathbb{Z}) .
$$

By [Ge5, Thm.6.2 and Prop.6.3], $\operatorname{coker}(\phi)$ is isomorphic to $H_{1}(\Gamma, \mathbb{Z})$, where $\Gamma$ is the dual graph associated with the curve $C$. We obtain an exact diagram

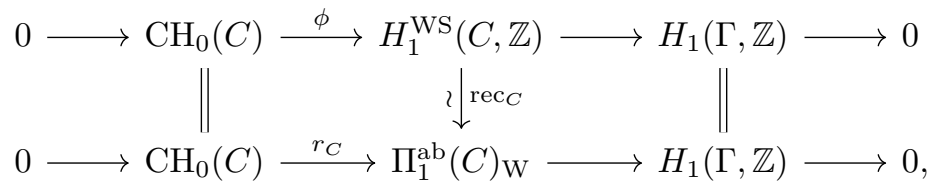

where, by Proposition 5.3, $r_{C}$ is the map which sends the class of a closed point to its Frobenius automorphism. Denoting by $\widehat{A}$ the profinite completion of an abelian group $A$, completion of the lower line yields the exact sequence

$$
0 \longrightarrow \widehat{\mathrm{CH}_{0}(C)} \longrightarrow \pi_{1}^{\mathrm{ab}}(C) \longrightarrow \widehat{H_{1}(\Gamma, \mathbb{Z})} \longrightarrow 0,
$$


which is the exact sequence of [KaSa, Prop. 1].

Finally, we consider the example of a nodal curve. Let $\widetilde{C}$ be a smooth, proper curve over $\mathbb{F}$ admitting two rational points $P, Q$, and let $C$ be the curve obtained by identifying $P$ and $Q$. We denote the image of $P$ and $Q$ in $C$ by $O$, i.e., we have an (abstract) blow-up square

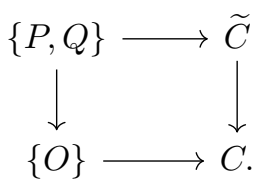

Let $D$ be a countable chain of copies $C_{i}, i \in \mathbb{Z}$, of $C$ with $P_{i} \in C_{i}$ identified with $Q_{i-1} \in C_{i-1}$ for all $i \in \mathbb{Z}$ :

$D$ :

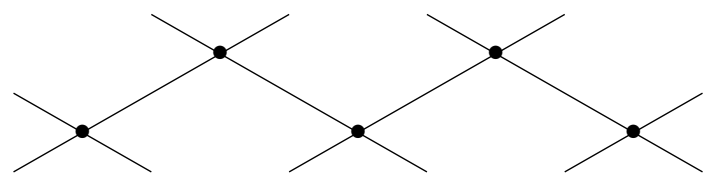

Translation gives a natural $\mathbb{Z}$-action on $D$ and $C$ is the quotient $D / \mathbb{Z}$ with respect to this action. In particular, we have a surjection $\Pi_{1}^{\mathrm{ab}}(C) \rightarrow \operatorname{Aut}_{C}(D)=\mathbb{Z}$. Inspecting the combinatorics of $D$, we see that

$$
\operatorname{ker}\left(\Pi_{1}^{\mathrm{ab}}(C) \rightarrow \mathbb{Z}\right) \cong \Pi_{1}^{\mathrm{ab}}(\widetilde{C}) /\left(\operatorname{Frob}_{P}-\operatorname{Frob}_{Q}\right) .
$$

Hence, we have an exact sequence

$$
0 \longrightarrow \Pi_{1}^{\mathrm{ab}}(\widetilde{C})_{W} /\left(\operatorname{Frob}_{P}-\operatorname{Frob}_{Q}\right) \longrightarrow \Pi_{1}^{\mathrm{ab}}(C)_{W} \longrightarrow \mathbb{Z} \longrightarrow 0 .
$$

In particular,

$$
\Pi_{1}^{\mathrm{ab}}(C)_{W} \cong \mathbb{Z} \oplus \mathbb{Z} \oplus \text { (finite) }
$$

as an abelian group. Applying Proposition 3.6 to the blow-up square (27) and using Examples 3.7 and 3.8, we obtain the exact sequence

$$
0 \longrightarrow \mathrm{CH}_{0}(\widetilde{C}) /([P]-[Q]) \longrightarrow H_{1}^{\mathrm{WS}}(C, \mathbb{Z}) \longrightarrow \mathbb{Z} \longrightarrow 0 .
$$

The reciprocity map $\mathrm{rec}_{C}$ induces an isomorphism between the exact sequences (29) and (28). The map on the left hand side is induced by the reciprocity map of the smooth curve $\widetilde{C}$, which sends $[x] \in \mathrm{CH}_{0}(\widetilde{C})$ to $\operatorname{Frob}_{x} \in \Pi_{1}^{\mathrm{ab}}(\widetilde{C})_{W}=\pi_{1}^{\mathrm{ab}}(\widetilde{C})_{W}$.

\section{References}

[DG] M. Demazure, P. Gabriel, Introduction to algebraic geometry and algebraic groups. Translated from the French by J. Bell. North-Holland Mathematics Studies, 39. North-Holland Publishing Co., Amsterdam-New York, 1980.

[Ge1] T. Geisser, Weil-étale cohomology over finite fields. Math. Ann. 330 (2004), no. $4,665-692$.

[Ge2] T. Geisser, Arithmetic cohomology over finite fields and special values of $\zeta$-functions. Duke Math. J. 133 (2006), no. 1, 27-57.

[Ge3] T. Geisser, On Suslin's singular homology and cohomology. Doc. Math. 2010, Extra volume: Andrei A. Suslin's sixtieth birthday, 223-249. 
[Ge4] T. Geisser, Duality via cycle complexes, Ann. of Math. (2) 172 (2010), no. $2,1095-1126$.

[Ge5] T. Geisser, Arithmetic homology and an integral version of Kato's conjecture. J. Reine Angew. Math. 644 (2010), 1-22.

[GS] T. Geisser, A. Schmidt, Tame Class Field Theory for Singular Varieties over Algebraically Closed Fields. Documenta Math. 21 (2016), 91-123.

[KaSa] K. Kato, S. Saito, Unramified class field theory of arithmetical surfaces. Ann. of Math. (2) 118 (1983), no. 2, 241-275.

[KeSa] M. Kerz, S. Saito, Cohomological Hasse principle and motivic cohomology for arithmetic schemes. Publ. Math. Inst. Hautes Etudes Sci. 115 (2012), 123-183.

[KeSc] M. Kerz, A. Schmidt, On different notions of tameness in arithmetic geometry. Math. Ann. 346 (2010), 641-668.

[Li] S. Lichtenbaum, Suslin homomology and Deligne 1-motives. In P. G. Goerss, J. F. Jardine (ed.): Algebraic K-theory and Algebraic Topology 1993, 189-196.

[MAS] K. Matsumi, K. Sato, M. Asakura, On the kernel of the reciprocity map of normal surfaces over finite fields. K-Theory 18 (1999), no. 3, 203-234.

[MVW] C. Mazza, V. Voevodsky and Ch. Weibel Lectures on Motivic Cohomology. Clay Monographs in Math. 2, AMS 2006.

[Mi] J. S. Milne, Etale cohomology. Princeton Mathematical Series, 33. Princeton University Press, Princeton, N. J. , 1980.

[Mu D. Mumford, Abelian varieties. Corrected reprint of the second (1974) edition. Tata Institute of Fundamental Research Studies in Mathematics, 5. Hindustan Book Agency, New Delhi, 2008.

[Sc] A. Schmidt, Singular homology of arithmetic schemes. Algebra \& Number Theory 1 (2007), no. 2, 183-222.

[SS] A. Schmidt, M. Spieß, Singular homology and class field theory of varieties over finite fields. J. Reine Angew. Math. 527 (2000), 13-36.

[SGA1] Revêtements étales et groupe fondamental. Lecture Notes in Mathematics 224, Springer-Verlag, Berlin, 1971. Séminaire de Géométrie Algébrique du Bois Marie 1960-1961 (SGA 1), Dirigé par A. Grothendieck. Augmenté de deux exposés de M. Raynaud.

[SGA3] Schémas en groupes. II: Groupes de type multiplicatif, et structure des schémas en groupes généraux. Lecture Notes in Mathematics 152, SpringerVerlag, Berlin-New York, 1970, Séminaire de Géométrie Algébrique du Bois Marie 1962-64 (SGA 3 II), Dirigé par M. Demazure et A. Grothendieck.

[SGA7] Groupes de monodromie en géométrie algébrique. II. Séminaire de Géométrie Algébrique du Bois-Marie 1967-1969 (SGA 7 II). Dirigé par P. Deligne et N. Katz. Lecture Notes in Mathematics, Vol. 340. SpringerVerlag, Berlin-New York, 1973.

[SV1] A. A. Suslin, V. Voevodsky, Singular homology of abstract algebraic varieties. Inv. Math. 123 (1996), no. 1, 61-94. 
[SV2] A. A. Suslin, V. Voevodsky Bloch-Kato conjecture and motivic cohomology with finite coefficients. The arithmetic and geometry of algebraic cycles (Banff, AB, 1998), 117-189, NATO Sci. Ser. C Math. Phys. Sci., 548, Kluwer Acad. Publ., Dordrecht, 2000.

Rikkyo University, Department of Mathematics, 3-34-1 Nishi-Ikebukuro, TOSHIMA-KU, TOKYO JAPAN 171-8501

E-mail address: geisser@rikkyo.ac.jp

Universität Heidelberg, Mathematisches Institut, Im Neuenheimer Feld 205, D-69120 Heidelberg, Deutschland

E-mail address: schmidt@mathi.uni-heidelberg.de 